\title{
Metallomics
}

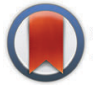

CrossMark <click for updates

Cite this: Metallomics, 2016, 8,422

Received 7th December 2015 Accepted 22nd January 2016

DOI: $10.1039 / c 5 m t 00312 a$

www.rsc.org/metallomics

\section{Behavior of platinum(Iv) complexes in models of tumor hypoxia: cytotoxicity, compound distribution and accumulation $\uparrow$}

\author{
Ekaterina Schreiber-Brynzak, ${ }^{a}$ Verena Pichler, ${ }^{a}$ Petra Heffeter, ${ }^{\text {bc }}$ Buck Hanson, ${ }^{\text {de }}$ \\ Sarah Theiner, ${ }^{\text {ac }}$ Irene Lichtscheidl-Schultz, ${ }^{f}$ Christoph Kornauth, ${ }^{9}$ Luca Bamonti, ${ }^{a}$ \\ Vineet Dhery, ${ }^{\text {bc }}$ Diana Groza, ${ }^{\text {bc }}$ David Berry, ${ }^{\text {de }}$ Walter Berger, ${ }^{\text {bc }}$ Mathea S. Galanski, \\ Michael A. Jakupec*ace and Bernhard K. Keppler ${ }^{\text {ace }}$
}

\begin{abstract}
Hypoxia in solid tumors remains a challenge for conventional cancer therapeutics. As a source for resistance, metastasis development and drug bioprocessing, it influences treatment results and disease outcome. Bioreductive platinum(Iv) prodrugs might be advantageous over conventional metal-based therapeutics, as biotransformation in a reductive milieu, such as under hypoxia, is required for drug activation. This study deals with a two-step screening of experimental platinum(Iv) prodrugs with different rates of reduction and lipophilicity with the aim of identifying the most appropriate compounds for further investigations. In the first step, the cytotoxicity of all compounds was compared in hypoxic multicellular spheroids and monolayer culture using a set of cancer cell lines with different sensitivities to platinum(॥) compounds. Secondly, two selected compounds were tested in hypoxic xenografts in SCID mouse models in comparison to satraplatin, and, additionally, (LA)-ICP-MS-based accumulation and distribution studies were performed for these compounds in hypoxic spheroids and xenografts. Our findings suggest that, while cellular uptake and cytotoxicity strongly correlate with lipophilicity, cytotoxicity under hypoxia compared to non-hypoxic conditions and antitumor activity of platinum(IV) prodrugs are dependent on their rate of reduction.
\end{abstract}

\section{Introduction}

Since its approval for cancer therapy in the late 1970s, cisplatin has been used so widely that it is often called "penicillin of cancer". The continued success of this chemotherapeutic drug inspired the further development of platinum(II) compounds for

\footnotetext{
${ }^{a}$ Institute of Inorganic Chemistry, University of Vienna, Waehringer Strasse 42, 1090 Vienna, Austria. E-mail: michael.jakupec@univie.ac.at; Tel: +431427752610

${ }^{b}$ Department of Medicine I, Institute of Cancer Research and Comprehensive Cancer Center, Medical University of Vienna, Borschkegasse 8a, 1090 Vienna, Austria ${ }^{2}$ Research Platform "Translational Cancer Therapy Research", University of Vienna, Waehringer Strasse 42, 1090 Vienna, Austria

${ }^{d}$ Department of Microbiology and Ecosystem Science, Division of Microbial Ecology, University of Vienna, Althanstrasse 14 (UZA I), 1090 Vienna, Austria

${ }^{e}$ Research Network Chemistry Meets Microbiology, University of Vienna, Althanstrasse 14 (UZA I), 1090 Vienna, Austria

${ }^{f}$ Core Facility Cell Imaging and Ultrastructure Research, University of Vienna, Althanstrasse 14 (UZA I), 1090 Vienna, Austria

${ }^{g}$ Clinical Institute of Pathology, Vienna General Hospital, Medical University of Vienna, Spitalgasse 23, 1090 Vienna, Austria

$\dagger$ Electronic supplementary information (ESI) available. See DOI: 10.1039/ c5mt00312a
}

anticancer therapy, generating hundreds of cisplatin analogs. Beside cisplatin, oxaliplatin and carboplatin are the only metalbased anticancer drugs widely established in clinical use, while others, such as picoplatin, are currently undergoing clinical trials. However, major limitations of platinum therapy, such as resistance development and dose-limiting side effects, remain. ${ }^{1-4}$ Some attempts to overcome the disadvantages of cisplatin treatment were made by exploring platinum(Iv) compounds. The most prominent representative of platinum(Iv)-based prodrugs, satraplatin, was undergoing different phase I, II, and III clinical trials as single and combination therapy for different cancer types, but most of the trials revealed moderate to low activity compared to standard treatment. ${ }^{5-7}$ Nevertheless, platinum(rv) complexes are promising anticancer agents, as they show high stability and bioavailability, allowing oral application and overcoming side effects such as nephrotoxicity. ${ }^{8,9}$ Platinum(rv) complexes have to be activated by reduction to release their active platinum(II) metabolites. Therefore, platinum(Iv) complexes, as bioreductive prodrugs, might target hypoxia and close the gap between classic metal-based and targeted therapies. ${ }^{10-12}$

Hypoxia, an important characteristic of progressive solid tumors, has been a challenge for conventional anticancer therapy 


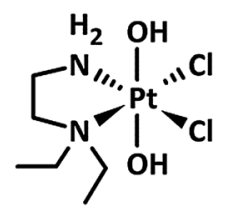

1

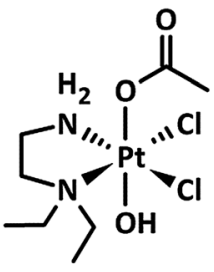

2

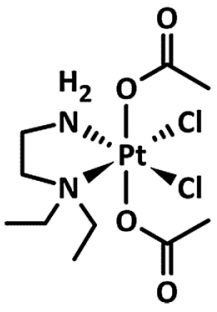

3

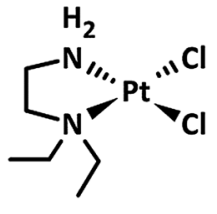

4

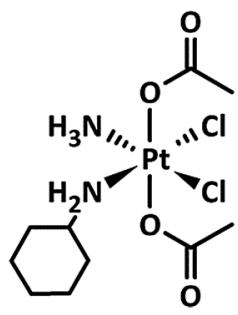

satraplatin

Fig. 1 Chemical structures of the platinum(IV) complexes 1-3 under investigation, including 4 as the main platinum(II) metabolite and the reference compound satraplatin.

for decades. ${ }^{13-17}$ As most chemotherapeutics, including platinum(II) drugs, target highly proliferating cancer cells, the strong quiescent cell fraction typically associated with hypoxia is hardly affected by many treatments. ${ }^{18,19}$ Additionally, necrotic and hypoxic regions generate inhomogeneities in tumor tissue, with changing physicochemical and biological properties, such as $\mathrm{pH}$ and $\mathrm{pO}_{2}$ gradients and different protein expression patterns. ${ }^{20}$ This bioreductive environment influences the stability of chemotherapeutic agents and can cause specific biotransformation of these drugs. In this case, platinum(Iv) complexes might have a substantial advantage over other therapeutic approaches, since they first have to be reduced for drug activation. Nevertheless, the activation of platinum(Iv) species in hypoxic tumors has barely been investigated so far.

Hall et al. and Mellor et al. suggested that platinum(Iv) complexes may be reduced to their active platinum(II) counterparts under conditions of solid tumors. ${ }^{21,23}$ Different small-molecular weight reducing agents, such as ascorbic acid, glutathione, or amino acids such as L-methionine and L-cysteine, but also proteins such as metallothioneins and albumin were discussed to be responsible for intra- as well as extracellular reduction in vivo and in vitro. ${ }^{22}$ However, the exact mechanism of platinum(Iv) reduction remains unknown and attempts to investigate the influence of the tumor micro-environment on platinum(Iv) complex stability were not fully conclusive. ${ }^{23}$ Nevertheless, the hypothesis of a possible reduction of platinum(Iv) compounds under hypoxic conditions in solid tumors continues to be discussed, whereas the confirmation of this hypothesis requires more experimental evidence.

This study specifically examines the influence of hypoxia on the activity of three investigational platinum(Iv) compounds, their main platinum(II) metabolite, and satraplatin as a reference compound (Fig. 1) in different in vivo and in vitro models of human cancer.

The investigated platinum(Iv) complexes have the same equatorial ligand sphere, whereas their axial ligands are different, with trans-dihydroxido, trans-hydroxidoacetato, and trans-diacetato ligands for 1, 2, and 3, respectively. The changes in the ligand sphere are associated with a different rate of reduction in the presence of ascorbic acid and glutathione, reduction potential and lipophilicity, whereas the possible platinum(II) metabolites and the stability are comparable. ${ }^{24}$ This set of physiochemical properties make these compounds very suitable as model complexes for investigating the impact of the rate of reduction on the antiproliferative activity of platinum(Iv) drugs under hypoxic conditions.

First, the investigational complexes 1-3 were tested in monolayer and hypoxic multicellular spheroid models to gain information about the dependence of their cytotoxic potencies on the cell culture conditions used. Subsequently, compounds 1 and 2 (performing best in hypoxic spheroids relative to monolayer cultures) were further tested in non-hypoxic spheroid models, hypoxic monolayer models and in a male SCID mouse model. Additionally, platinum distribution and cellular accumulation studies in spheroids gave further insights into the behavior of the compounds and their mode of action.

\section{Results and discussion}

\section{Characterization of hypoxia within spheroids}

Spheroids are in vitro tumor models, which share some characteristics with in vivo tumors, such as proliferation and nutrition gradients, chronic hypoxia and necrosis. Hypoxic regions and the necrotic core of spheroids with diameters of $>400 \mu \mathrm{m}$ (further referred to as "hypoxic spheroids" or "hypoxic 3D") were visualized previously by using HIF1 $\alpha$ immunofluorescence and PI staining, respectively. ${ }^{25}$ The obtained images displayed massive necrosis within the spheroid core and revealed that a region of mild hypoxia starts already at a depth as low as $30 \mu \mathrm{m}$ beneath the spheroid surface. Spheroids with diameters of $<200 \mu \mathrm{m}$, however, showed no evidence of hypoxia or necrosis ${ }^{25}$ and are therefore referred to as "non-hypoxic spheroids or non-hypoxic 3D".

Characterization was now continued with regard to the hypoxic environment within live spheroids. For this purpose, oxygen distribution within $>400 \mu \mathrm{m}$ large spheroids was measured by an invasive microelectrode technique. Attempts to measure oxygen tension in live spheroids with microelectrodes published by other authors faced some technical problems and raised issues for critical discussion. For example, the steady-state measurements reported by Mueller-Klieser et al. took $1 \mathrm{~min}$ per step, with a total measuring time of $20 \mathrm{~min}$ per spheroid. ${ }^{26}$ In this time period, it is likely that passive diffusion affects the data. To bypass this problem, we used novel technical equipment, which allows 
A

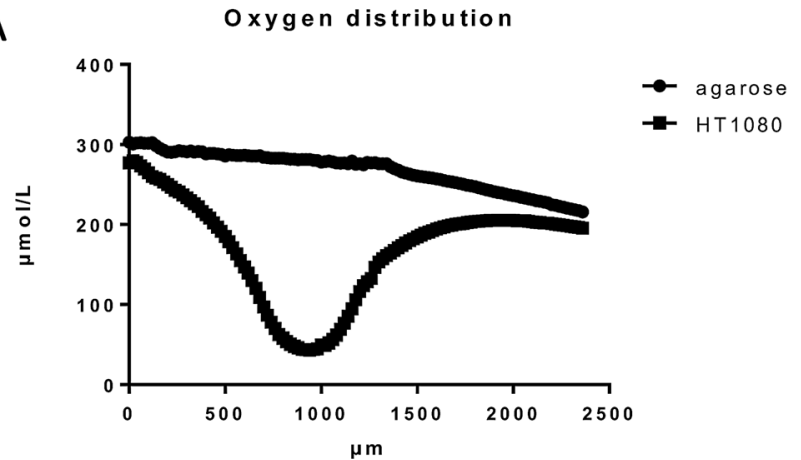

B

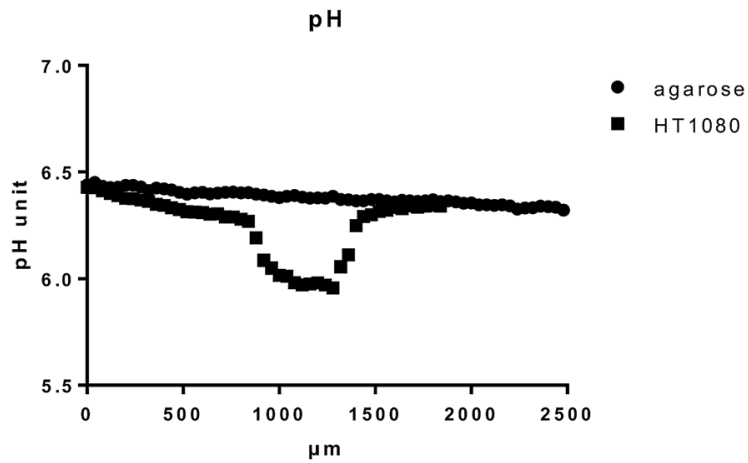

C

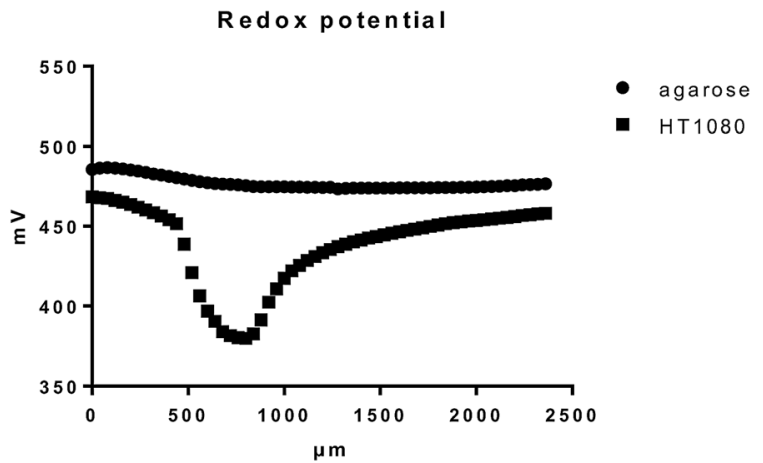

Oxygen distribution

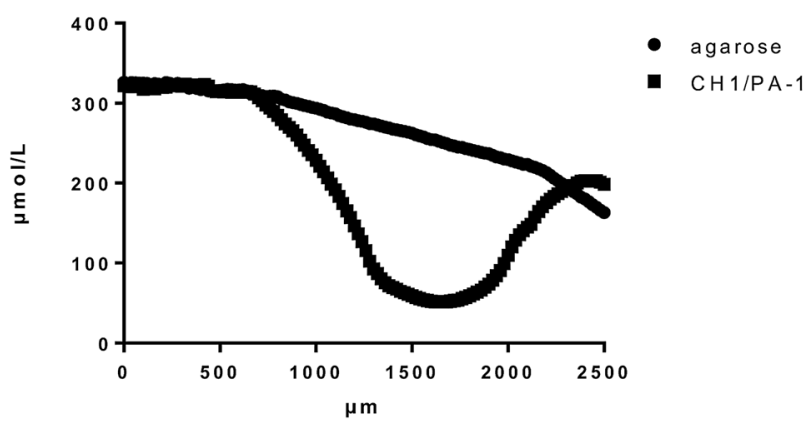

$\mathrm{pH}$

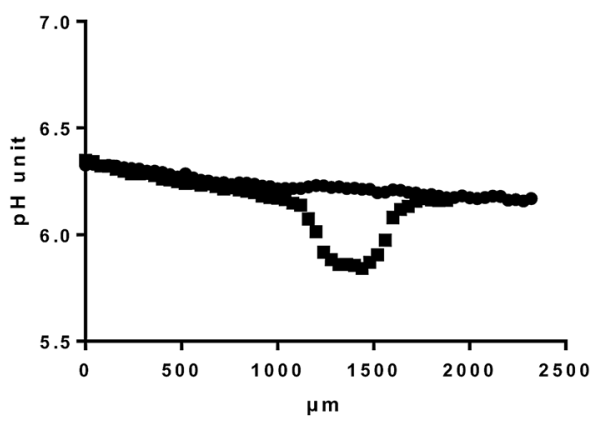

- agarose

- $\mathrm{CH} 1 / \mathrm{PA}-1$

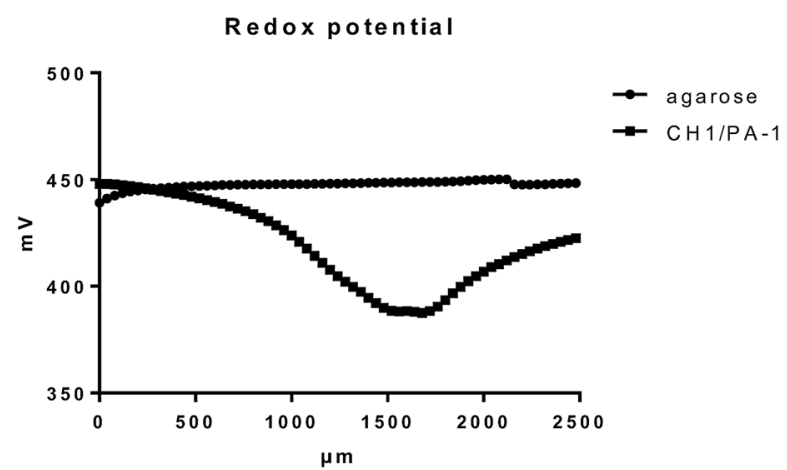

Fig. 2 Physicochemical gradients through agarose-embedded HT1080 and CH1/PA-1 multicellular spheroids (> $400 \mu \mathrm{m}$ diameter) in comparison to agarose medium distant from the spheroid, measured by microelectrodes and exemplified by representative samples. (A) Oxygen distribution (measured in $20 \mu \mathrm{m}$ intervals), (B) pH (40 $\mu \mathrm{m}$ intervals), (C) redox potential (40 $\mu \mathrm{m}$ intervals). Profile depths are relative to the agarose surface, whereby spheroid location relative to the agarose surface varies.

the completion of the measurement within 4 s per step, with a total measurement time of about 1.5 min per spheroid.

Our results featured oxygen gradient towards the necrotic core of the spheroid, with the lowest oxygen tension in the spheroid center, as shown here in HT1080 and CH1/PA-1 spheroids (Fig. 2A and ESI $\dagger$ ). These results excellently correspond with the data published by other authors. ${ }^{26,27}$ Comparable patterns have been monitored for all investigated cell lines and samples (at least 9 samples per cell line); but small variations in absolute values depending on the cell line and therefore on the tightness of the spheroids were observed as well as individual differences between spheroids of the same cell line. Fig. 2 shows exemplary results for HT1080 and CH1/PA-1 spheroids. The experimental setup required sample immobilization and preservation of humidity and temperature; for this purpose, samples were embedded in agarose. A markedly decreasing oxygen level was noticeable already in the surrounding of the spheroid, but not in the agarose profile measurement without spheroids (used here as negative control), which only showed a very slow decrease of oxygen in deeper layers of agarose.

Hypoxia is usually accompanied by a significant drop in $\mathrm{pH}$ (acidosis), which is regularly observed in solid tumors ${ }^{28,29}$ and multicellular spheroids. ${ }^{30} \mathrm{As} \mathrm{pH}$ is a major factor in drug stability for most chemotherapeutics, we investigated if and to which extent a $\mathrm{pH}$ gradient is measurable in our samples by using the microelectrode method. The results showed a $\mathrm{pH}$ gradient 
towards the spheroid center following the oxygen distribution pattern but hardly extending to the surroundings of the spheroid (Fig. 2B and ESI $\dagger$ ). The observed $\mathrm{pH}$ difference of $\sim 0.2 \mathrm{pH}$ units between the spheroid surface and the spheroid center corresponds very well with the results published previously by other authors. ${ }^{30} \mathrm{pH}$ profiles give a rough idea about the dimension of the spheroids $(>400 \mu \mathrm{m})$, whereas oxygen gradients are flatter and extend much more into the surrounding agarose, most likely due to diffusion following continued oxygen consumption by the viable spheroids.

Furthermore, hypoxia was reported to cause a significant decrease of the reduction potential in cancer cells grown in monolayers. ${ }^{31}$ While it is to be expected that massive hypoxia in a spheroid core will also cause a significant decrease of the reductive potential, measurements have not been reported so far. To evaluate the redox potential within the used spheroid models, microelectrode measurements in live spheroids were conducted. Our data demonstrate a considerable drop of the redox potential towards the spheroid center similar to the oxygen and $\mathrm{pH}$ distribution patterns (Fig. 2C and ESI $\dagger$ ). Unfortunately, the experimental setup does not allow a distinction between intracellular and extracellular regions. Overall, the decreased oxygen level, $\mathrm{pH}$ and reduction potential emphasize the presence of a reductive milieu within the spheroids, most pronounced in the necrotic core (Fig. 2 and ESI $\dagger$ ).

\section{Antiproliferative activity}

Investigation of the antiproliferative activity in cancer cell lines is the first crucial step in exploring the anticancer potential of experimental compounds. Therefore, we studied the cytotoxicity of compounds 1-4 and satraplatin and compared them to oxaliplatin (l-OHP) (data published previously ${ }^{25}$ ) in different hypoxic and nonhypoxic cell culture models using cell lines with varying sensitivity for platinum(II) drugs, including $\mathrm{CH} 1 / \mathrm{PA}-1$ ovarian teratocarcinoma (most sensitive), HCT116 colon cancer (moderately sensitive), and HT1080 fibrosarcoma (insensitive) cells. In the first step, the $\mathrm{IC}_{50}$ values were determined in hypoxic spheroids and non-hypoxic monolayer cultures by means of the Alamar Blue assay. In the non-hypoxic monolayer culture, all complexes showed the highest activity in the sensitive cell line CH1/PA-1 with $\mathrm{IC}_{50}$ values mostly in the low micromolar range, whereas the lowest activity was observed in the resistant cell line HT1080. In general, the activity of the complexes increases in the following order in all three cell lines: $\mathbf{1}<\mathbf{4}<\mathbf{2}<\mathbf{3}<$ satraplatin $<l$-OHP, indicating that the reference compounds satraplatin and oxaliplatin are the most cytotoxic in monolayer culture. This order of investigational compounds 1-3 corresponds very well with the lipophilicity of these substances, with $\mathbf{1}$ being the least lipophilic and $\mathbf{3}$ the most lipophilic. These data are in very good accordance with the previously published results (compare ref. 24). The slightly divergent values in the cell line CH1/PA-1 compared to those published in ref. 38 are probably caused by the application of different reagents for staining of viable cells (MTT vs. Alamar Blue).

In the hypoxic spheroid (3D) model (spheroids with diameters of $>400 \mu \mathrm{m}$, positive for HIF1 $\alpha$ and PI staining), cytotoxicity manifested differently to extents depending on the compound.
In the CH1/PA-1 spheroid model, the activity of compounds 1-4 shifted to even lower $\mathrm{IC}_{50}$ values, whereas satraplatin and oxaliplatin were negatively affected. These results underline the sensitivity of the cell line $\mathrm{CH} 1 / \mathrm{PA}-1$, as in the less sensitive cell lines HT1080 and HCT116 most $\mathrm{IC}_{50} \mathrm{~S}$ were shifted to higher values, with the only exception of 1 in HT1080 cells. When comparing the results obtained in the 3D CH1/PA-1 model and the rate of reduction, it becomes obvious that the cytotoxic potency of the slowly reducing substance 1 (factor 6.8) benefits most, whereas the faster reducing 2 and 3 (factors 1.6 and 1.7, respectively) show only a minor activation under hypoxia. Though in the less sensitive cell lines HT1080 and HCT116 all cytotoxic potencies are negatively affected, factors decrease in a similar order: 1 (factors 1.3 and 0.6 ) $>2$ (factors 0.7 and 0.3 ) $>3$ (factors 0.4 and 0.1 ).

Surprisingly, the platinum(II) species (4) showed a 1.6-fold activation in the CH1/PA-1 spheroid model, whereas the cytotoxicity of satraplatin and oxaliplatin is diminished. As this compound contains platinum already in the more reactive oxidation state + II, the observed activation of complex 4 in the CH1/PA-1 model cannot be explained by reduction. But remarkably, the platinum(II) reference compound oxaliplatin (l-OHP) did not exhibit a comparable activation. The most important structural differences between oxaliplatin and $\mathbf{4}$ are the leaving groups: one chelating oxalate group in contrast to two chlorides, respectively. These initial ligands have to be released by aquation prior to binding of the platinum(II) center to DNA. Therefore, the rate of aquation influenced by these ligands may be one possible explanation for the activation of complex 4. This hypothesis is supported by the previously published results by Knox et al. that cisplatin has a 112-fold faster hydrolysis than oxaliplatin. ${ }^{32}$ Furthermore, $\mathrm{pH}$ and hence the $\mathrm{pH}$-gradient in 3D-models are crucial for the hydrolysis process and the subsequent DNA binding of platinum(II) species, as it was reported that at $\mathrm{pH}>8$ the aqua species is transformed into the less reactive hydroxido counterpart, whereas a $\mathrm{pH}<8$ increases the binding kinetics. ${ }^{33}$ Nevertheless, additional investigations will be necessary to clarify the relationship of hypoxia and platinum(II) compounds.

Since oxaliplatin, as a platinum(II) reference compound, showed up to 10 -fold increased $\mathrm{IC}_{50}$ values when comparing the cytotoxicity in $3 \mathrm{D}$ with that in $2 \mathrm{D}$ experiments (Table 1), we hypothesized that the observed increase in cytotoxic activity of platinum(Iv) prodrugs might reflect their activation by reduction. To support this consideration, compounds $\mathbf{1}$ (lowest reduction rate and lipophilicity) and 2 (intermediate reduction rate and lipophilicity), as the best performing compounds according to the order of activation in hypoxic spheroids of all investigated cell lines, were chosen for further investigations in non-hypoxic spheroids and hypoxic monolayer culture as well as in hypoxic xenografts.

Unexpectedly, hypoxia in monolayer culture does not elicit the activation of compounds in the sensitive cell line CH1/PA-1, while in the less sensitive cell lines HT1080 and HCT116 IC $_{50}$ values are increased, comparable to those obtained in hypoxic spheroid culture (Fig. 3 and Table 2). These results underline the difference between hypoxia in monolayer culture and spheroids, with monolayers being appropriately supplied with nutrients, 
Table 1 Cytotoxicity of complexes 1-4, satraplatin and oxaliplatin (l-OHP) in hypoxic spheroid (3D) models and non-hypoxic monolayer (2D) models, determined by means of the Alamar Blue assay (exposure time $96 \mathrm{~h}$ ). Values are means \pm SDs from at least three independent experiments performed in triplicate

\begin{tabular}{|c|c|c|c|c|c|c|c|c|c|}
\hline & \multicolumn{9}{|l|}{$\underline{\mathrm{IC}_{50}[\mu \mathrm{M}]}$} \\
\hline & CH1/PA-1 2D & CH1/PA-1 3D & $2 \mathrm{D} / 3 \mathrm{D}$ & HT1080 2D & HT1080 3D & $2 \mathrm{D} / 3 \mathrm{D}$ & HCT116 2D & HCT116 3D & $2 \mathrm{D} / 3 \mathrm{D}$ \\
\hline 1 & $6.2 \pm 0.6$ & $0.90 \pm 0.13$ & 6.8 & $96 \pm 2$ & $77 \pm 11$ & 1.3 & $42 \pm 9$ & $65 \pm 10$ & 0.6 \\
\hline 3 & $1.6 \pm 0.1$ & $1.0 \pm 0.2$ & 1.7 & $9.8 \pm 2.1$ & $21 \pm 5$ & 0.4 & $4.0 \pm 0.4$ & $29 \pm 7$ & 0.1 \\
\hline 4 & $4.6 \pm 0.9$ & $1.9 \pm 0.1$ & 2.4 & $35 \pm 8$ & $47 \pm 5$ & 0.7 & $35 \pm 4$ & $71 \pm 6$ & 0.5 \\
\hline Satra-Pt & $0.24 \pm 0.04$ & $0.40 \pm 0.01$ & 0.5 & $5.1 \pm 0.3$ & $7.8 \pm 1.1$ & 0.6 & $2.7 \pm 0.6$ & $6.0 \pm 1.3$ & 0.4 \\
\hline$I-\mathrm{OHP}{ }^{a}$ & $0.20 \pm 0.01$ & $1.4 \pm 0.2$ & 0.2 & $1.7 \pm 0.1$ & $18 \pm 3$ & 0.09 & $0.57 \pm 0.10$ & $1.6 \pm 0.2$ & 0.4 \\
\hline
\end{tabular}

A

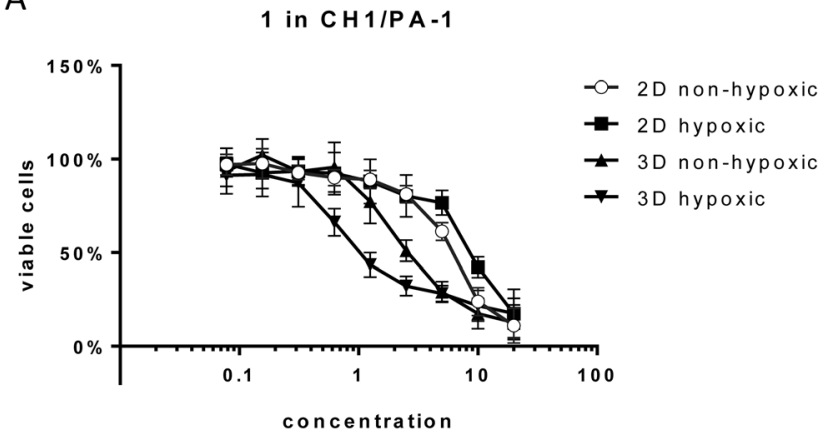

B

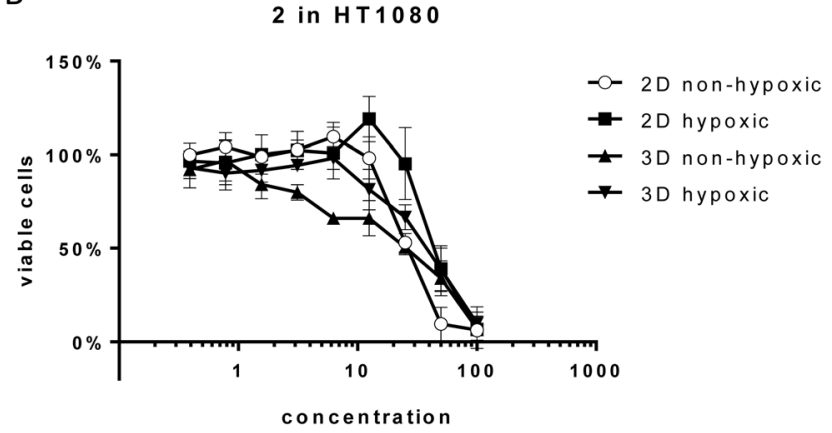

Fig. 3 Cytotoxicity of compounds 1 and 2 in hypoxic versus nonhypoxic spheroids and hypoxic versus non-hypoxic monolayer culture. (A) Concentration-effect curves of 1 in CH1/PA-1 cells. (B) Concentrationeffect curves of $\mathbf{2}$ in HT1080 cells. Values are means \pm SDs from at least three independent experiments performed in triplicate.

showing an early response to the lack of oxygen, but no signs of necrosis or apoptosis (results not shown) and other $\mathrm{pH}$ distribution and protein expression patterns. ${ }^{34,35}$

For 1 in the non-hypoxic spheroid model (spheroids with diameters of $<200 \mu \mathrm{m}$, negative for HIF1 $\alpha$ and PI staining) of $\mathrm{CH} 1 / \mathrm{PA}-1$, the $\mathrm{IC}_{50}$ value is intermediate between those obtained in monolayer culture and hypoxic spheroids (hypoxic $2 \mathrm{D}>$ nonhypoxic $2 \mathrm{D}>$ non-hypoxic $3 \mathrm{D}>$ hypoxic $3 \mathrm{D}$ ) (Fig. 3). For 2, $\mathrm{IC}_{50}$ values in hypoxic and non-hypoxic $\mathrm{CH} 1 / \mathrm{PA}-1$ spheroids are comparable, indicating the activation of both compounds in both spheroid models compared to monolayer culture (Table 2). The same applies for $\mathbf{1}$ and $\mathbf{2}$ in non-hypoxic HT1080 spheroids, with the $\mathrm{IC}_{50}$ value in the latter being comparable to that in the hypoxic spheroid model. In HCT116 cells, however, IC $_{50}$ values in non-hypoxic spheroids are comparable to those obtained in monolayer culture. In general, these observations correspond to the results obtained in hypoxic spheroids, showing again the high sensitivity of $\mathrm{CH} 1 / \mathrm{PA}-1$ cells compared to HCT116 and HT1080 cells and a similar order of activation: $\mathbf{1} \geq 2$.

\section{Drug accumulation and distribution studies}

Poor drug uptake and penetration were discussed as factors for the decreased activity of different compounds in spheroid models. ${ }^{36,37}$ To shed light on the relationship between drug penetration and different cytotoxicities for the platinum complexes under investigation, platinum distribution studies were performed for 1, 2, and satraplatin in hypoxic HT1080 spheroids by using LA-ICP-MS. All compounds showed comparable penetration of the hypoxic HT1080 spheroids with Pt accumulation hot spots in the necrotic spheroid core (Fig. 4A-C), indicating a high affinity of the platinum complexes to hypoxic regions and underlining the possible targeting effect to these regions. Furthermore, we clearly showed that drug penetration is not a reason for differences in the cytotoxicity of platinum(Iv) complexes in the HT1080 spheroid model.

Additionally, the data of total drug accumulation into hypoxic spheroids showed that $\mathbf{3}$ is taken up more effectively by all three cell lines than the other platinum compounds (Table 3). This finding correlates with the cytotoxicity data (Table 1), as compound 3 has the lowest $\mathrm{IC}_{50}$ values in spheroids as well as in monolayer culture. In general, the amount of platinum accumulation correlates perfectly with the lipophilicity of the compounds, as the order of lipophilicity is equivalent to the order of increased accumulation, with 3 (most lipophilic) $>2>1$ (least lipophilic) (Table 3). However, neither lipophilicity nor accumulation corresponds to the activation in hypoxic spheroids. Although compound 1 showed 7-fold activation and compound 2 2-fold activation in hypoxic $\mathrm{CH} 1 / \mathrm{PA}-1$ spheroids, compound 1 accumulates less effectively than compound $\mathbf{2}$. In particular, $\mathbf{3}$, with the highest accumulation, is comparably active in hypoxic CH1/PA-1 spheroids as 1.

In general, accumulation studies in hypoxic spheroids of all studied cell lines showed the following order of accumulation: $3>2>4>1$. IC $_{50}$ values, obtained in hypoxic HT1080 $(3>2 / 4>1)$, HCT116 $(3>2>4 / 1)$ and CH1/PA-1 $(3 / 1>2 / 4)$ spheroids, in general correspond to the order of accumulation, in line with compound 3 as the most cytotoxic one. The order of 
Table 2 Cytotoxicity of compounds 1 and $\mathbf{2}$ in hypoxic versus non-hypoxic spheroids as well as hypoxic and non-hypoxic monolayer culture

\begin{tabular}{|c|c|c|c|c|c|c|c|c|}
\hline \multirow[b]{2}{*}{ Cell line } & \multicolumn{8}{|l|}{$\underline{\mathrm{IC}_{50}[\mu \mathrm{M}]}$} \\
\hline & $2 \mathrm{D}$ & 2D hypox. & $3 \mathrm{D}$ & 3D hypox. & $2 \mathrm{D}$ & 2D hypox. & $3 \mathrm{D}$ & 3D hypox. \\
\hline CH1/PA-1 & $6.2 \pm 0.6$ & $8.6 \pm 1.0$ & $2.6 \pm 0.4$ & $0.9 \pm 0.1$ & $3.1 \pm 0.6$ & $2.2 \pm 0.9$ & $1.5 \pm 0.2$ & $1.9 \pm 0.2$ \\
\hline HCT116 & $42 \pm 9$ & $74 \pm 5$ & $27 \pm 3$ & $66 \pm 10$ & $14 \pm 2$ & $38 \pm 1$ & $17 \pm 2$ & $52 \pm 12$ \\
\hline HT1080 & $96 \pm 22$ & $87 \pm 9$ & $43 \pm 11$ & $77 \pm 11$ & $26 \pm 2$ & $45 \pm 8$ & $27 \pm 5$ & $40 \pm 8$ \\
\hline
\end{tabular}

A

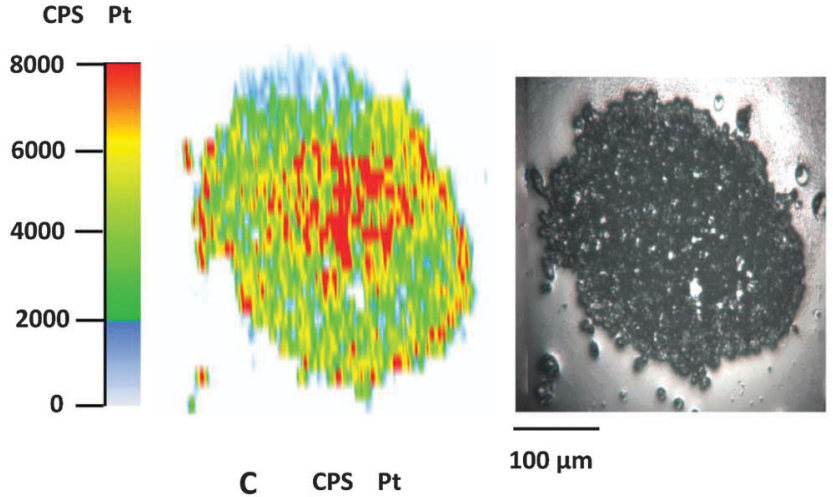

B CPS Pt
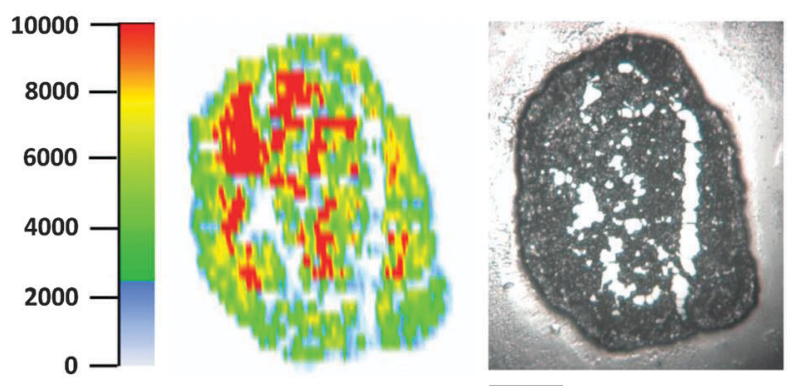

$100 \mu \mathrm{m}$

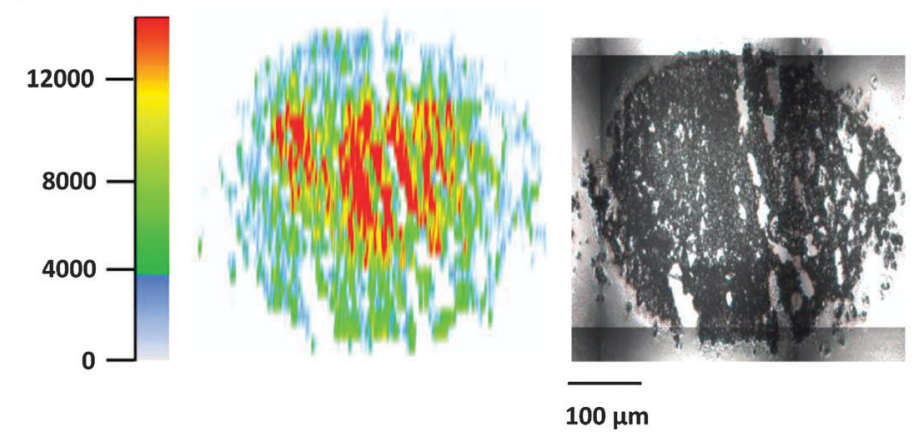

Fig. 4 Platinum distribution in hypoxic HT1080 spheroids upon treatment with (A) compound 1, (B) compound $\mathbf{2}$ and (C) satraplatin. Laser ablation-ICP-MS measurements were performed using $20 \mu \mathrm{m}$ thin cryosections.

Table 3 Platinum accumulation in hypoxic spheroid models treated with 1-4 in relation to rate of reduction and lipophilicity of the compound. Values are means \pm SDs from at least three independent experiments performed in triplicates

\begin{tabular}{lcccll}
\hline \multicolumn{5}{c}{ Platinum accumulation in ng per spheroid } \\
\cline { 2 - 6 } & CH1/PA-1 & HT1080 & HCT116 & $\begin{array}{l}\text { Reduction } \\
\text { half time }^{a}\end{array}$ & $\begin{array}{l}\text { Lipophilicity } \\
\log k_{\mathrm{w}}{ }^{b}\end{array}$ \\
\hline 1 & $0.31 \pm 0.07$ & $0.13 \pm 0.03$ & $0.16 \pm 0.03$ & $\sim 15 \mathrm{~h}$ & 0.51 \\
2 & $0.26 \pm 0.06$ & $0.45 \pm 0.06$ & $0.47 \pm 0.08$ & $\sim 5 \mathrm{~h}$ & 1.05 \\
3 & $1.3 \pm 0.3$ & $1.9 \pm 0.1$ & $1.9 \pm 0.3$ & $\sim 15 \mathrm{~min}$ & 1.60 \\
4 & $0.45 \pm 0.12$ & $0.34 \pm 0.06$ & $0.40 \pm 0.04$ & - & - \\
${ }^{a}$ & Obtained from ref. $24 .{ }^{b}$ Obtained from ref. 38.
\end{tabular}

activation in hypoxic spheroids if compared with monolayer culture, however, shows no correlation with accumulation data. The order of activation in hypoxic spheroids (HT1080 $(1>2 / 4>3)$; HCT116 $(\mathbf{1}>\mathbf{4}>\mathbf{2}>3)$; CH1/PA-1 $(\mathbf{1}>\mathbf{4}>\mathbf{2} / \mathbf{3}))$ rather underlines that compound 3 with the highest accumulation shows the lowest activation under hypoxia, while compound 1 with the lowest accumulation is activated under hypoxia to the highest extent. These results suggest that activity under hypoxia is not highly dependent on drug accumulation and lipophilicity, but seems to be dependent on the rate of reduction of the applied platinum(rv) compounds.

\section{Animal studies}

1, 2, and satraplatin (as a reference compound) were further investigated in human xenografts using SCID mice. The less sensitive HT1080 cell line was chosen as the most suitable for xenograft experiments, due to decreased or comparable $\mathrm{IC}_{50}$ values of both compounds in the hypoxic spheroid model and good applicability in the mouse model. Compounds were administered in equimolar doses ( $8.3 \mathrm{mg}$ for compound $1,9.1 \mathrm{mg}$ for compound 2 and $10 \mathrm{mg}$ for satraplatin) on days $4,7,11$, and 14 . Satraplatin caused only a transient growth delay, with reduced tumor burden on days 11 and $12\left({ }^{* *} p<0.01\right.$ two-way ANOVA, Fig. 5A). However, this was followed by tumor regrowth resulting in nearly no difference in the tumor burden compared to control animals at day 15 . With regard to our novel platinum(Iv) drugs, the slowly reducing complex 1 did not display any 
A

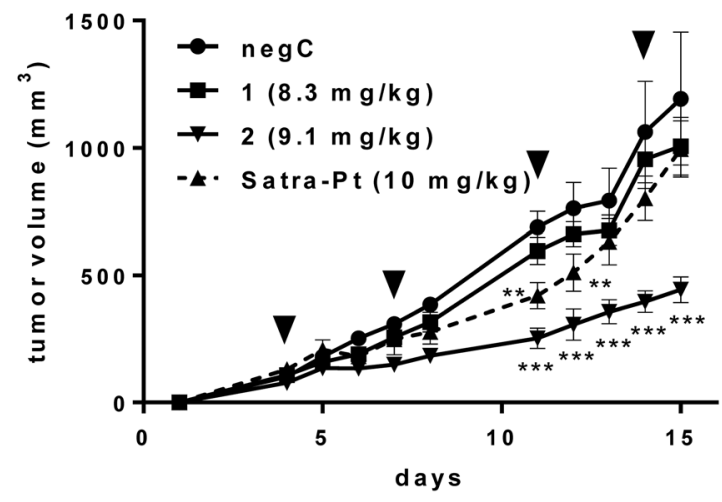

C

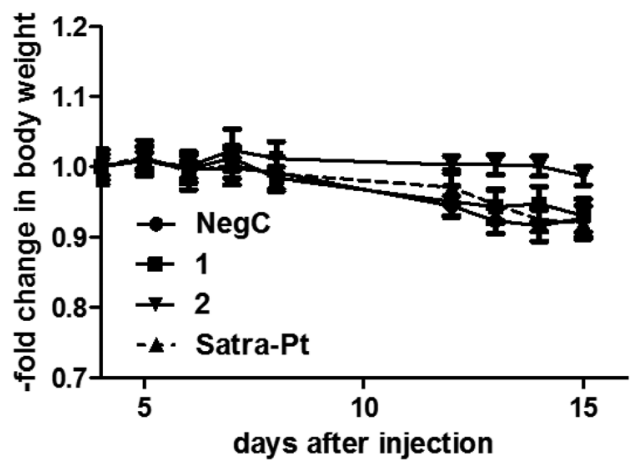

B

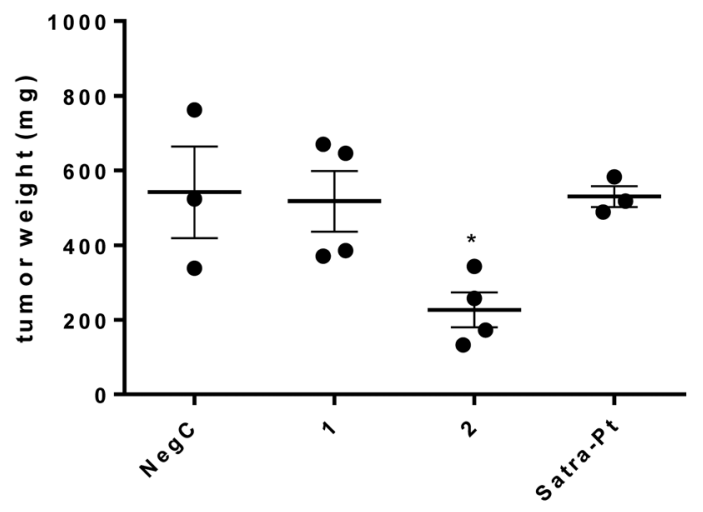

D

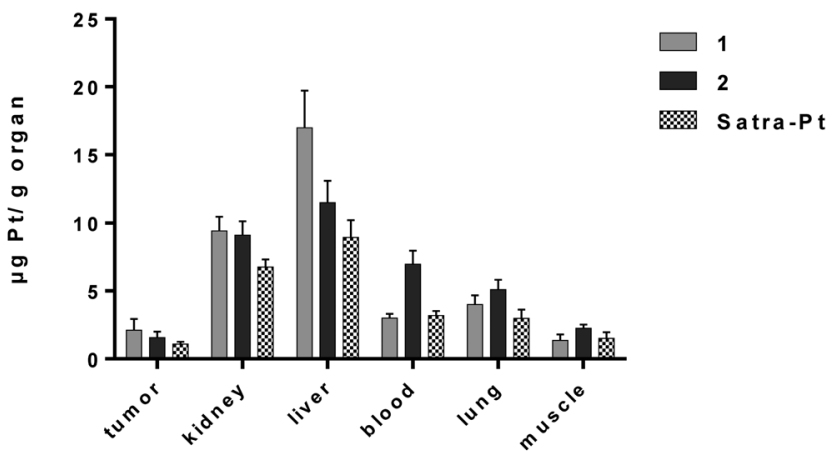

Fig. 5 (A) In vivo activity of equimolar doses of 1, 2, and satraplatin in male SCID mice with subcutaneous tumors of human HT1080 cells ( $n=4$, treatment i.p. on days 4, 7, 11, 14 (indicated by black arrows)), ${ }^{* *} p<0.01$ and ${ }^{* * *} p<0.001$ by two-way ANOVA. (B) Tumor weight compared to negative control for all treatment groups at day 15, significantly different to control: ${ }^{*} p<0.05$ by one-way ANOVA. In the negative control and the satraplatin group, tumor weights of only 3 animals could be determined due to tumor ulceration in one animal each on day 13 and 14, respectively. (C) Body weight from all treatment groups. (D) Platinum concentration (mean \pm SD) from 1, 2 and satraplatin in mouse tissues, blood pellet and tumor, collected at day 15 , determined by ICP-MS.

anticancer activity. In contrast, the easiest reducible substance 2 significantly reduced tumor growth $(* * * p<0.001$ by two-way ANOVA) from day 11 (Fig. 5A) and caused significantly reduced tumor burden ( $p<0.05$, two-way ANOVA) at day 15 (Fig. 5B). This is also reflected in body weight, as the rapid tumor growth of HT1080 cells is associated with a slow decline of body weight, which is not observed in 2-treated animals bearing reduced tumor burden (Fig. 5C).

Comparing the order of in vivo activity $(1<$ satraplatin $<2)$ with the physicochemical properties of the substances, no relation to the lipophilicity is evident, whereas the role of the rate of reduction seems consistent, with an intermediate reduction rate being highly favorable for in vivo activity. Furthermore, platinum levels in the tumor tissue are not the solely decisive factor, as $\mathbf{2}$ is not accumulated to the highest degree, but rather intermediate between those of 1 and satraplatin (Fig. 5C). All compounds accumulate in the kidney, suggesting renal elimination, but, remarkably, the least lipophilic compound $\mathbf{1}$ has the highest accumulation in the liver. An increased platinum concentration from 2 is found in the blood stream, suggesting a higher retention in the circulatory system compared to $\mathbf{1}$ and satraplatin.

\section{Platinum distribution in tumor sections analyzed by LA-ICP-MS}

Bioimaging by LA-ICP-MS has been shown to be advantageous to assess the platinum distribution in histologically heterogeneous structures, compared to measurements of the average platinum concentration in tissues by ICP-MS. HT1080 tumors of SCID mice after equimolar treatment with compounds $\mathbf{1}, \mathbf{2}$ or satraplatin were prepared for LA-ICP-MS measurements to determine the platinum accumulation at a microscopic level. The platinum distribution was correlated with histology (visualized by an H\&E stain of a consecutive slide) and with the hypoxic regions which were co-localized by pimonidazole staining in confocal microscopy. Simultaneous detection of necrotic regions was not possible due to fixation steps required for pimonidazole staining.

In general, a heterogeneous platinum distribution was observed in the ablated tumor sections after treatment with the respective platinum compounds. The areas of platinum enrichment (indicated by red color) most likely correspond to the localization of blood (micro-)vessels (Fig. 6B and E) and to loose, soft tissue (which is only sparsely infiltrated with tumor cells) as well as to striated muscle of the treated mouse (Fig. 6C-E). 
A CPS Pt
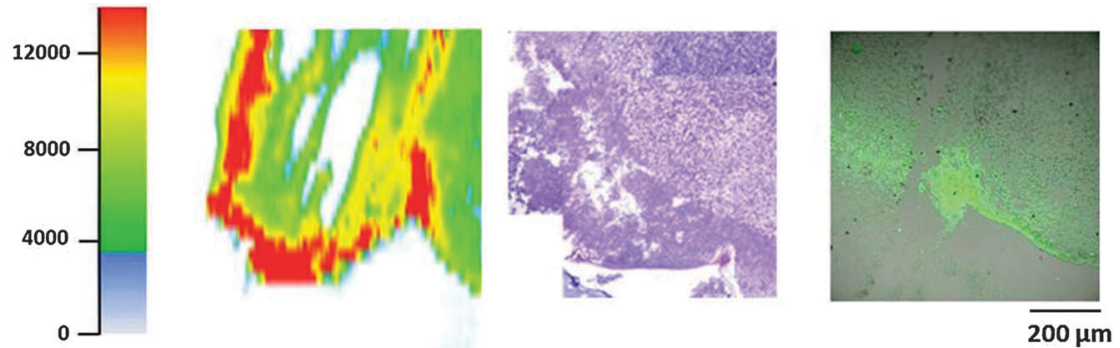

B CPS Pt

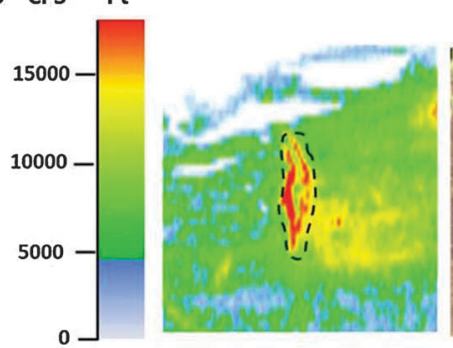

D CPS Pt

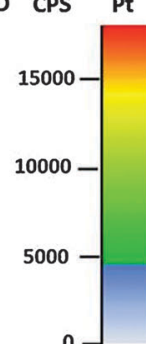

E CPS Pt

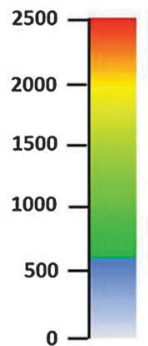

F CPS Pt
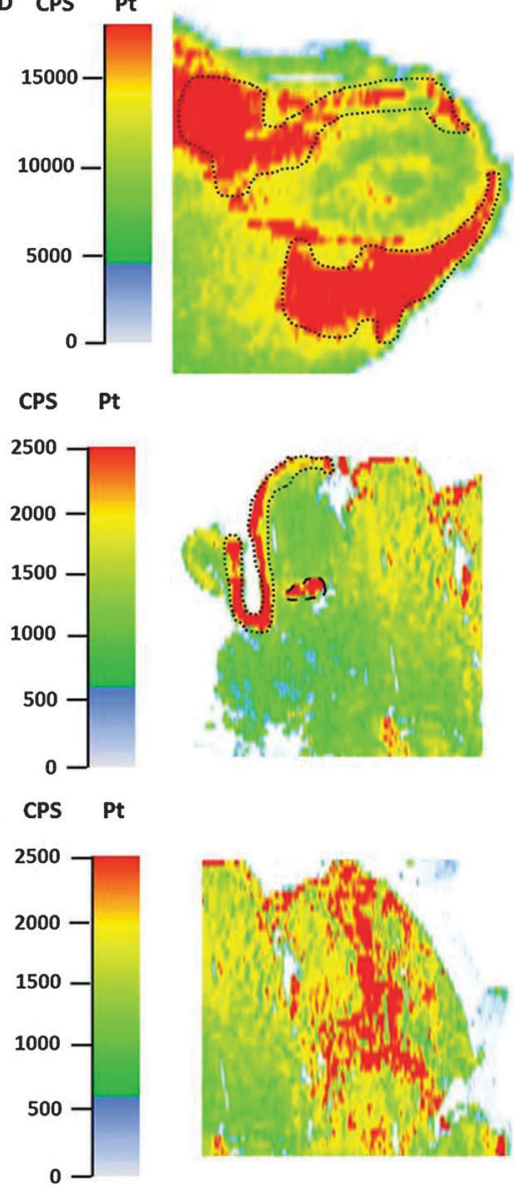

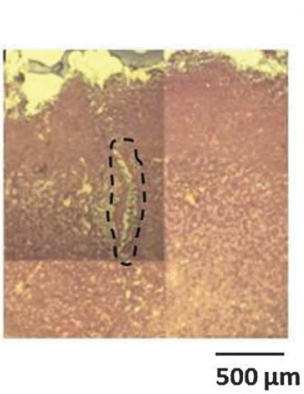

c
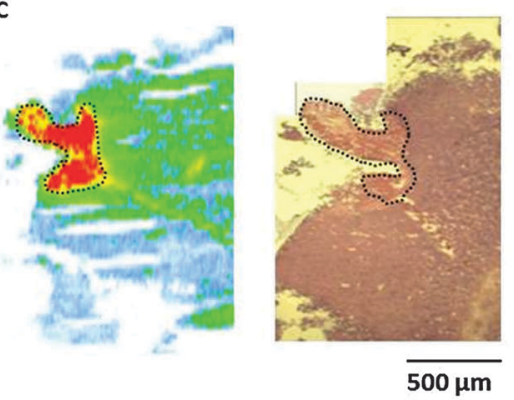
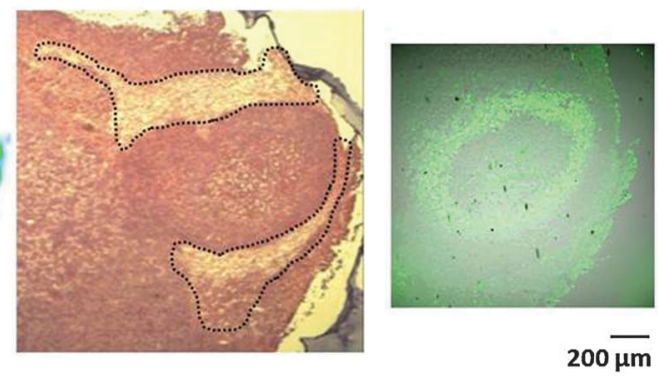

$200 \overline{\mu \mathrm{m}}$

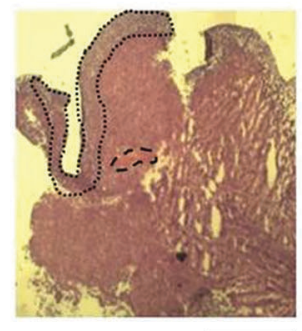

$500 \mu \mathrm{m}$
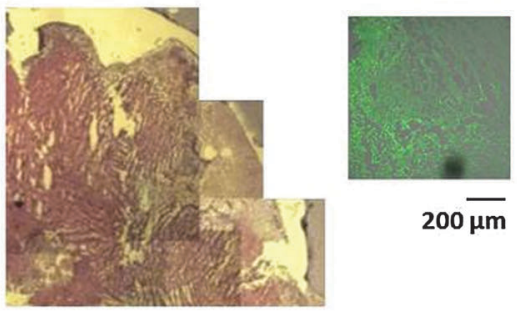

Fig. 6 Platinum distribution in HT1080 tumor sections measured by LA-ICP-MS. Detailed views of ablated tumor samples after treatment with compounds $1(A)$, compound $\mathbf{2}(B-D)$ and satraplatin (E and F); laser platinum image (left), H\&E-stained section (middle) and hypoxic regions, visualized by pimonidazole staining (right). The dotted spots in the laser image and H\&E-stained slides correspond to loose, connective tissue with sparsely scattered tumor cells. The dashed lines indicate the presence of a blood (micro-)vessel.

Malignant tissue, which is composed of denser packed tumor cells, in contrast, generally exhibited lower platinum levels. These results are in accordance with previous LA-ICP-MS studies on platinum complexes in tumors of CT26-colon cancer bearing mice. ${ }^{39}$ 
In detail, for the tumor sections after treatment with compound 1 (Fig. 6A) and satraplatin (Fig. 6F) a correlation with platinum enrichment and hypoxic regions was observed. The ablated tumor sample after treatment with compound 2 revealed the presence of a tumor nodule (confirmed by the underlying histology). Again, areas of loose, soft tissue surrounding the tumor nodule comprised increased platinum amounts (Fig. 6D). Still, platinum was also highly concentrated in the tumor nodule, where the histology indicates the presence of a central necrotic region. Interestingly, pimonidazole co-localized here with a rim of platinum enrichment around the central necrosis. These results are in agreement with the laser ablation data of the HT1080 spheroids with increased platinum levels in the core and reveal the ability of platinum species to penetrate into these regions in vitro as well as in vivo.

\section{Conclusion}

Hypoxia-induced activation of platinum(Iv) drugs in solid tumors is an attractive strategy for targeting anticancer therapies. Therefore, investigations were performed on the relationship between physicochemical properties of three platinum(Iv) complexes, their platinum(II) counterpart and satraplatin as a reference complex and the cell biological responses in non-hypoxic and hypoxic $2 \mathrm{D}$ as well as $3 \mathrm{D}$ tumor cell culture models. Subsequently, the findings were underpinned by in vivo experiments in a SCID male mouse model.

Although a strong correlation could be found between lipophilicity, drug accumulation and 2D and 3D cytotoxicity, the extent of drug accumulation is hardly related to the activation under hypoxia in hypoxic spheroids and the SCID mouse model. Rather, activity seems to be dependent on the rate of reduction of these complexes, although data suggest an activation even for the platinum(II) species 4 in one of the three spheroid models. Complex 1 with the lowest rate of reduction is least active in all tested cell lines and settings, whereas the activities of the fastest reducing compound 3 seem to be affected by too fast biotransformation in hypoxic 3D models, especially in less sensitive cell lines. On the other hand, compound 2, with an intermediate lipophilicity and reduction rate, seems to have optimal properties for high efficacy. This complex elicited a significant inhibition of tumor growth in vivo superior to 1 and satraplatin. To the best of our knowledge, we are the first to show the activation of platinum(Iv) complexes in a 3D $\mathrm{CH} 1 / \mathrm{PA}-1$ in vitro model compared to the corresponding 2D model. These findings indicate an advantage of these platinum(Iv) compounds over satraplatin.

\section{Material and methods}

\section{Cell lines and culture conditions}

The human cell line CH1 (identified via STR profiling as PA-1 ovarian teratocarcinoma cells by Multiplexion; see also ref. 40) was kindly provided by Lloyd R. Kelland (CRC Centre for Cancer Therapeutics, Institute of Cancer Research, Sutton, UK), and the colon cancer cell line HCT116 (ATCC ${ }^{\circledR}$ CCL-247 ${ }^{\mathrm{TM}}$ ) was purchased from American Type Culture Collection (ATCC). These cell lines were grown in MEM (supplemented with 10\% heat-inactivated fetal bovine serum (Life Technologies, Austria, Vienna), $1 \%$ L-glutamine, $1 \%$ sodium pyruvate, $1 \%$ non-essential amino acids solution (all from Sigma Aldrich, Austria, Vienna)). Human HT1080 (ATCC ${ }^{\circledR}$ CCL-121 ${ }^{\mathrm{TM}}$ ) fibrosarcoma cells and CCD-18Co normal colon fibroblasts were obtained from ATCC (CRL1459) and maintained in MEM (Sigma) supplemented with 10\% FCS. Monolayer cultures of all cell lines were grown in cell culture treated $75 \mathrm{~cm}^{2}$ flasks (Starlab, Hamburg, Germany).

\section{Spheroid culture}

For spheroid production, CH1/PA-1, HCT116, and HT1080 cells were harvested from culture flasks by trypsinization and seeded in MEM using non-cell culture treated round bottom 96-well plates (VWR) in densities of $1 \times 10^{4}$ (CH1/PA-1), $2 \times 10^{3}$ (HCT116), and $8 \times 10^{3}$ (HT1080) viable cells per well, respectively, for spheroids with $>400 \mu \mathrm{m}$ diameter 7 days prior to treatment; and in densities of $1 \times 10^{3}$ (CH1/PA-1), $0.3 \times 10^{3}$ (HCT116), $0.3 \times 10^{3}$ (HT1080) viable cells per well, respectively, for spheroids with $<200 \mu \mathrm{m}$ diameter 3 days prior to treatment. To minimize evaporation, the outermost wells were not used for spheroid production and filled with $200 \mu \mathrm{L}$ of PBS instead. Cultures were maintained at $37{ }^{\circ} \mathrm{C}$ in a humidified atmosphere containing $95 \%$ air and $5 \% \mathrm{CO}_{2}$.

\section{Compounds}

Compounds (1, 2, 3, 4, satraplatin and oxaliplatin (l-OHP)) have been synthesized at the Institute of Inorganic Chemistry, University of Vienna, according to literature procedures. ${ }^{38}$ All platinum(Iv) compounds were dissolved in MEM to stock solutions of $2 \mathrm{mM}$ and then diluted in MEM to the required concentrations. Cisplatin and 4 were dissolved shortly before usage in MEM to a stock concentration of $0.4 \mathrm{mM}$ and diluted further in MEM.

\section{Alamar Blue assay}

For tests with monolayers, CH1/PA-1, HCT116, and HT1080 cells were harvested from culture flasks by trypsinization and seeded in MEM into 96-well microculture plates (Falcon) $24 \mathrm{~h}$ prior to treatment in densities of $1 \times 10^{3}$ (CH1/PA-1), $2 \times 10^{3}$ (HCT116), $3 \times 10^{3}$ (HT1080) viable cells per well, respectively. For tests in $3 \mathrm{D}$ cultures, spheroids with the required diameter were treated directly in culture plates.

For both monolayer and spheroid treatment, stock solutions of the test compounds were prepared and diluted stepwise to obtain a dilution series. $100 \mu \mathrm{L}$ of dilution were added to each well, and plates were incubated for $96 \mathrm{~h}$ at $37{ }^{\circ} \mathrm{C}$ and $5 \% \mathrm{CO}_{2}$. A fresh solution of $440 \mu \mathrm{M}\left(\approx 110 \mu \mathrm{g} \mathrm{mL}{ }^{-1}\right)$ resazurin sodium salt (Sigma Aldrich) in PBS was prepared and $20 \mu \mathrm{L}$ of it were added to each well. Plates were stained at $37{ }^{\circ} \mathrm{C}$ and $5 \% \mathrm{CO}_{2}$ for $4 \mathrm{~h}$ (monolayer experiments) or overnight (spheroid experiments).

\section{ICP-MS}

Chemicals. Milli-Q water (18.2 $\mathrm{M} \Omega \mathrm{cm}$, Milli-Q Advantage, Darmstadt, Germany) was used for all dilutions for ICP-MS measurements. Nitric acid ( $\geq 65 \%$, p.a., Fluka, Buchs, Switzerland) 
Table 4 Instrumental parameters for (LA-)ICP-MS measurements

\begin{tabular}{|c|c|c|}
\hline & ICP-MS & LA-ICP-MS \\
\hline RF power $[\mathrm{W}]$ & 1500 & 1350 \\
\hline Cone material & Nickel & Nickel \\
\hline Carrier gas $\left[1 \mathrm{~min}^{-1}\right]$ & 0.90 & 1.10 \\
\hline Make up gas $\left[1 \min ^{-1}\right]$ & 0.20 & - \\
\hline Plasma gas $\left[1 \mathrm{~min}^{-1}\right]$ & 15 & 15 \\
\hline Monitored isotopes & ${ }^{185} \mathrm{Re},{ }^{194} \mathrm{Pt},{ }^{195} \mathrm{Pt}$ & ${ }^{195} \mathrm{Pt}$ \\
\hline Dwell time $[\mathrm{s}]$ & 0.3 & 0.1 \\
\hline Number of replicates & 10 & 1 \\
\hline
\end{tabular}

was further purified in a quartz sub-boiling point distillation unit (Milestone-MLS GmbH, Leutkirch, Germany). Platinum and rhenium standards for ICP-MS measurements were purchased from CPI International (Amsterdam, The Netherlands). Tissue-Tek medium (Sakura Finetek, The Netherlands) was used for embedding the cryosections. All other reagents and solvents were obtained from commercial sources and were used without further purification.

ICP-MS measurements. The total platinum content in spheroids and in mouse tissues was determined by using an ICP-quadrupole MS instrument Agilent 7500ce (Agilent Technologies, Waldbronn, Germany). The ICP-MS instrument was equipped with a CETAC ASX-520 autosampler (Nebraska, USA) and a MicroMist nebulizer

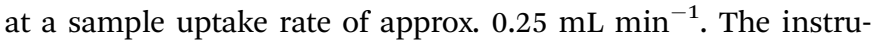
ment was tuned daily and rhenium served as internal standard for platinum to account for instrumental fluctuations and matrix effects. The instrumental parameters are summarized in Table 4. The Agilent MassHunter software package (Workstation Software, Version B.01.01, 2012) was used for data processing.

In the case of bioimaging by LA-ICP-MS, data were recorded by using a Triple Quadrupole ICP-MS Agilent 8800 (Agilent Technologies, Tokyo, Japan) using the instrumental parameters given in Table 4.

Sample preparation for accumulation studies (ICP-MS). Spheroids were treated with $20 \mu \mathrm{M}$ of compounds 1-4 for $4 \mathrm{~h}$, then harvested and transferred into $1.5 \mathrm{~mL}$ Eppendorf tubes (10 spheroids with an average diameter of $450 \mu \mathrm{m}$ per tube). After $3 \times$ washing with $1 \mathrm{~mL}$ PBS, spheroids were lysed with $400 \mu \mathrm{L}$ $\mathrm{HNO}_{3}$ for $1 \mathrm{~h}$ and filled up with Milli-Q water to a final volume of $8 \mathrm{~mL}$. The total platinum content was determined with ICP-MS using the instrumental parameters summarized in Table 4.

Determination of the platinum concentration in mouse tissues by ICP-MS. Digestion of mouse tissue samples (tumor, kidney, liver, lung, muscle) as well as blood pellets was performed with sub-boiled nitric acid by using a microwave system Discover SP-D (CEM Microwave Technology, Germany). The following microwave parameters were used: temperature: $200{ }^{\circ} \mathrm{C}$; ramp time: $4 \mathrm{~min}$; hold time: $6 \mathrm{~min}$; maximal power: $300 \mathrm{~W}$. Digested samples were diluted with Milli-Q water resulting in nitric acid concentrations lower than 3\% and platinum concentrations lower than $15 \mathrm{ng} \mathrm{g}^{-1}$. The total platinum content was determined with ICP-MS using the instrumental parameters given in Table 4 .

Bioimaging in multicellular spheroids and mouse tumor samples by LA-ICP-MS. After compound treatment with 1-5 $\mu \mathrm{M}$ (sub-cytotoxic concentrations) for $96 \mathrm{~h}$, spheroids were harvested and transferred into $1.5 \mathrm{~mL}$ Eppendorf tubes (10-20 spheroids per tube) and washed $3 \times$ with PBS. After the last washing step, PBS was removed completely and Eppendorf tubes were carefully filled up with $0.5-1 \mathrm{~mL}$ Tissue Tek (Sakura) prior to freezing at $-80{ }^{\circ} \mathrm{C}$. Spheroids were marked with $1 \mu \mathrm{L}$ of trypan blue. Subcutaneous xenografts were removed and frozen at $-80^{\circ} \mathrm{C}$. For LA-ICP-MS measurements, samples were cryosectioned into slices of $20 \mu \mathrm{m}$ thickness by using a cryotom (Microm HM 550, Thermo Fischer), placed onto glass slides and air dried. A consecutive slice of $5 \mu \mathrm{m}$ of the respective organ was stained with hematoxylin-eosin (H\&E) and used for histological evaluation.

A Nd:YAG solid state laser (NWR 213, ESI, Fremont, CA, USA) at a wavelength of $213 \mathrm{~nm}$ was used to obtain the spatially resolved distribution of platinum in spheroids and tumor sections. An optical sample map of the region of interest was generated prior to the measurement. The output laser energy was tuned separately for every sample in order to ensure complete ablation of the sample material. Ablation was performed using parallel line scans and a spacing of $10 \mu \mathrm{m}$ between the lines. For tumor sections, a frequency of $10 \mathrm{~Hz}$, a spot size of $70 \mu \mathrm{m}$ and a scan speed of $40 \mu \mathrm{m} \mathrm{s}^{-1}$ was used, whereas for spheroids the laser parameters were adapted to $20 \mathrm{~Hz}$, a spot size of $10 \mu \mathrm{m}$ and a scan speed of $10 \mu \mathrm{m} \mathrm{s}^{-1}$. The ablated sample material was transferred to the ICP-MS instrument with helium (quality 5.0) at a flow rate of $400 \mathrm{~mL} \mathrm{~min}^{-1}$. Data were recorded by using an ICP-MS Agilent 8800 and the instrumental parameters summarized in Table 4. The software Igor Pro (Wavemetrics, Igor Pro 6.34A) together with its add-on Iolite (Iolite Version 2.5) was used for further data processing and generation of platinum distribution maps. The data reduction scheme 'Trace_Elements' including blank subtraction and no smoothing of the visualization was applied according to the manual by the authors (Iolite User Manual, Version 2.0). ${ }^{41}$ The aspect ratio of the image was set according to the dimensions of the ablated area in order to obtain accurately shaped pictures.

\section{Hypoxia detection}

Tumor slices were fixed with acetone and stained according to the Hypoxyprobe ${ }^{\mathrm{TM}}-1$ Kit (hpi) protocol. Alexa Flour 647-labeled secondary antibody (Cell Signaling, Frankfurt am Main, Germany) diluted 1:1000 in PBST was used for fluorescence detection in the confocal microscope Leica CLSM (Leica Microsystems, Wetzlar, Germany). As the spheroid sections did not stand fixation due to poor cellular cohesion and wash steps required for Hypoxyprobe ${ }^{\mathrm{TM}}-1$ Kit application, whole spheroids were stained with HIF-1 alpha antibody as described previously. ${ }^{25}$

\section{Hypoxic chamber}

For hypoxia induction in monolayer culture, cells were seeded into 96-well plates and allowed to attach for $24 \mathrm{~h}$ prior to treatment in a hypoxic chamber (C-chamber and ProOx C21, BioSpherix) with the oxygen level lowered to $0.5 \%$ by displacement with nitrogen. Treatment of cells was performed for $96 \mathrm{~h}$ in the hypoxic chamber with $0.5 \%$ oxygen. 


\section{Animal experiments}

Six- to 8-week-old male CB-17 scid/scid (SCID) mice were purchased from Harlan Laboratories (San Pietro al Natisone, Italy). The animals were kept in a pathogen-free environment and every procedure was done in a laminar airflow cabinet. The experiments were done according to the regulations of the Ethics Committee for the Care and Use of Laboratory Animals at the Medical University Vienna (proposal number BMWF-66.009/0084-II/3b/2013), The U.S. Public Health Service Policy on Human Care and Use of Laboratory Animals as well as the United Kingdom Coordinating Committee on Cancer Prevention Research's Guidelines for the Welfare of Animals in Experimental Neoplasia.

For the local tumor growth experiments, HT1080 cells $\left(1 \times 10^{6}\right)$ were injected subcutaneously into the right flank. Animals were randomly assigned to treatment groups and therapy was started when tumor nodules were $\sim 100 \mathrm{~mm}^{3}$. Animals were treated with 1 (i.p. $8.3 \mathrm{mg} \mathrm{kg}^{-1}$ dissolved in 5\% DMSO), 2 (i.p. $9.1 \mathrm{mg} \mathrm{kg}^{-1}$ dissolved in $5 \%$ DMSO), or satraplatin (i.p. $10 \mathrm{mg} \mathrm{kg}^{-1}$ dissolved in $5 \%$ DMSO) on days $4,7,11$, and 14 . Animals in the control group received water intraperitoneally. Animals were controlled for distress development every day and tumor size was assessed regularly by caliper measurement. Tumor volume was calculated by using the formula: (length $\times$ width $\left.^{2}\right) / 2$.

At day 15 , animals received pimonidazole $\left(60 \mathrm{mg} \mathrm{kg}^{-1}\right.$ i.p. dissolved in $0.9 \% \mathrm{NaCl}$ ) and were sacrificed $30-60 \mathrm{~min}$ later. For this purpose, the mice were anesthetized and blood was collected by heart punctuation.

\section{Oxygen and redox microsensor measurements}

For microsensor measurements, spheroids were washed once in sterile filtered PBS followed by resuspension in $1 \%$ agarose prepared by using $1 \times$ PBS. The suspended spheroids were aliquoted onto a pre-heated $\left(37^{\circ} \mathrm{C}\right)$ Petri dish containing a $1 \mathrm{~cm}$ layer of PBS-agarose (1\%). Samples were maintained at $37{ }^{\circ} \mathrm{C}$ during microsensor measurements in a circulating waterbath (ThermoScientific SC 100-S19T, Karlsruhe, Germany).

Oxygen and redox potential profiles were measured by using microsensors (10 $\mu \mathrm{m}$ tip diameter; Unisense, Aarhus, Denmark). Microsensor calibration and measurements were implemented according to the manufacturer instructions via the software interface (SensorTrace Suite, Unisense Aarhus, Denmark) provided with the microsensors. Microsensors were calibrated by using external standards from two-point standard curves generated prior to measurements.

For oxygen measurements, a saturated atmospheric oxygen reading was obtained from a vigorously aerated volume of water at $20{ }^{\circ} \mathrm{C}$. After $5 \mathrm{~min}$ of aeration, the bubbling was stopped and replicate measures were recorded. The saturated oxygen concentration was calculated by using the microsensor software based upon the solution temperature and salinity. An anoxic solution was prepared containing $0.1 \mathrm{M}$ sodium ascorbate in $0.1 \mathrm{M} \mathrm{NaOH}$ and replicate measures were performed. Oxygen distribution was measured with the step size of $20 \mu \mathrm{m}$ and total measuring time per step was $4 \mathrm{~s}$.

The redox microsensor was calibrated with saturated solutions of quinhydrone $\left(10 \mathrm{~g} \mathrm{~L}^{-1}\right)$ in $\mathrm{pH}$ standards of 4.0 and 7.0.
Redox measurements were performed by using an $\mathrm{Ag} / \mathrm{AgCl}$ reference electrode (REF 321 Radiometer Analytical, Denmark). Depth profiles were collected by fixing the microsensors in a 2D micro profiling system (Unisense, Aarhus, Denmark) and controlled by using the SensorTrace Suite software. Redox measurements were performed with a step size of $30 \mu \mathrm{m}$ and total measuring time per step was $14 \mathrm{~s}$.

\section{Statistics}

All animal data are expressed as mean \pm SEM. Results were analyzed and illustrated by using GraphPad Prism (version 5; GraphPad Software, San Diego, CA). Statistical analyses were performed by using one- and two-way ANOVA with drug treatment and time as independent variables, and conducted with Bonferroni post-tests to examine the differences between the different drug treatment regimens and the diverse responses. The statistical significance is either described in the respective figure legends, or indicated with asterisks $\left({ }^{*} p<0.05\right.$; ${ }^{* *} p<0.01$; ${ }^{* *} p<0.001$ ).

\section{Acknowledgements}

These studies were performed in cooperation with different institutes and groups, as well as single persons; therefore, we would like to thank all partners for cooperation and support of this study. First of all, we want to acknowledge financial support provided by the Vienna Science and Technology Fund (Project LS12-001) and FWF (projects P26127-B20 and P26603), which made our work possible. We are also grateful to Sushilla van Schoonhoven and Gerhard Zeitler for technical support. This project was carried out in the surrounding of COST Action CM1105.

\section{References}

1 L. Kelland, Nat. Rev. Cancer, 2007, 7(8), 573-584.

2 X. Yao, K. Panichpisal, N. Kurtzman and K. Nugent, Am. J. Med. Sci., 2007, 334(2), 115-124.

3 M. H. Hanigan and P. Devarajan, Cancer Ther., 2003, 1, 47-61.

4 H. Choy, Expert Rev. Anticancer Ther., 2006, 6(7), 973-982.

5 H. Choy, C. Park and M. Yao, Clin. Cancer Res., 2008, 14(6), 1633-1638.

6 J. W. Smith 2nd, K. J. McIntyre, P. V. Acevedo, C. A. Encarnacion, K. L. Tedesco, Y. Wang, L. Asmar and J. A. O'Shaughnessy, Breast Cancer Res. Treat., 2009, 118(2), 361-367.

7 R. W. Gregg, J. M. Molepo, V. J. Monpetit, N. Z. Mikael, D. Redmond, M. Gadia and D. J. Stewart, J. Clin. Oncol., 1992, 5, 795-803.

8 M. D. Galsky, L. H. Camacho, E. G. Chiorean, D. Mulkerin, D. S. Hong, W. K. Oh and D. F. Bajorin, Ann. Oncol., 2012, 23(4), 1037-1044.

9 G. Doshi, G. Sonpavde and C. N. Sternberg, Expert Opin. Drug Metab. Toxicol., 2012, 8(1), 103-111.

10 M. D. Galsky, S. Seng, L. H. Camacho, E. G. Chiorean, D. Mulkerin, D. S. Hong, W. K. Oh and D. F. Bajorin, Clin. Genitourin. Cancer, 2011, 9(1), 27-30. 
11 E. Wexselblatt and D. Gibson, J. Inorg. Biochem., 2012, 117, 220-229.

12 S. Vouillamoz-Lorenz, T. Buclin, F. Lejeune, J. Bauer, S. Leyvraz and L. A. Decosterd, Anticancer Res., 2003, 23(3C), 2757-2765.

13 L. H. Gray, A. D. Conger, M. Ebert, S. Hornsey and O. C. Scott, Br. J. Radiol., 1953, 26, 638-648.

14 Radiobiology for the radiologist, ed. E. J. Hall, Lippincott, Philadelphia PA, 4th edn, 1994.

15 R. P. Hill, The basic science of oncology, 1992, pp. 259-275.

16 T. G. Graeber, C. Osmanian, T. Jacks, D. E. Housman, C. J. Koch and S. W. Lowe, et al., Nature, 1996, 379, 88-91.

17 C. Y. Kim, M. H. Tsai, C. Osmanian, T. G. Graeber, J. E. Lee and R. G. Giffard, et al., Cancer Res., 1997, 57, 4200-4204.

18 R. E. Durand and P. L. Olive, Methods Cell Biol., 2001, 64, 211-233.

19 A. M. Shannon, D. J. Bouchier-Hayes, C. M. Condron and D. Toomey, Cancer Treat. Rev., 2003, 29(4), 297-307.

20 G. Helmlinger, F. Yuan, M. Dellian and R. K. Jain, Nat. Med., 1997, 3, 177-182.

21 M. D. Hall, H. R. Mellor, R. Callaghan and T. W. Hambley, J. Med. Chem., 2007, 50(15), 3403-3411.

22 N. Graf and J. Stephen, Adv. Drug Delivery Rev., 2012, 64(11), 993-1004.

23 H. R. Mellor, S. Snelling, M. D. Hall, S. Modok, M. Jaffar, T. W. Hambley and R. Callaghan, Biochem. Pharmacol., 2005, 70(8), 1137-1146.

24 V. Pichler, S. Göschl, E. Schreiber-Brynzak, M. A. Jakupec, M. Galanski and B. K. Keppler, Metallomics, 2015, 7, 1078-1090.

25 E. Schreiber-Brynzak, E. Klapproth, C. Unger, I. LichtscheidlSchultz, S. Göschl, S. Schweighofer, R. Trondl, H. Dolznig, M. A. Jakupec and B. K. Keppler, Invest. New Drugs, 2015, 33(4), 835-847.

26 W. F. Mueller-Klieser and R. M. Sutherland, Br. J. Cancer, 1982, 45(2), 256-264.
27 H. Acker, J. Carlsson, W. Mueller-Klieser and R. M. Sutherland, Br. J. Cancer, 1987, 56(3), 325-327.

28 J. Chiche, M. C. Brahimi-Horn and J. Pouysségur, J. Cell. Mol. Med., 2010, 14(4), 771-794.

29 L. E. Gerweck and K. Seetharaman, Cancer Res., 1996, 56(6), 1194-1198.

30 A. Görlach and H. Acker, Molecular Basis of Disease, 1994, 1227(3), 105-112.

31 J. Jiang, C. Auchinvole, K. Fisher and C. J. Campbell, Nanoscale, 2014, 6, 12104-12110.

32 R. J. Knox, F. Friedlos, D. A. Lydall and J. J. Roberts, Cancer Res., 1986, 46(4 Pt 2), 1972-1979.

33 T. Zimmerman, J. Leszczynski and J. V. Burda, J. Mol. Model., 2011, 17(9), 2385-2393.

34 S. Walenta, J. Doetsch, W. Mueller-Klieser and L. A. KunzSchughart, J. Histochem. Cytochem., 2000, 48, 509-522.

35 C. Mischiati, B. Ura, L. Roncoroni, L. Elli, C. Cervellati, M. Squerzanti, D. Conte, L. Doneda, P. Polverino de Laureto, G. de Franceschi, R. Calza, C. A. Barrero, S. Merali, C. Ferrari, C. M. Bergamini and E. Agostinelli, PLoS One, 2015, 10(3), e0118906.

36 D. J. Kerr, T. E. Wheldon, S. Hydns and S. B. Kaye, Xenobiotica, 1988, 18(6), 641-648.

37 G. Mehta, A. Y. Hsiao, M. Ingram, G. D. Luker and S. Takayama, J. Controlled Release, 2012, 164(2), 192-204.

38 V. Pichler, S. Göschl, S. M. Meier, A. Roller, M. A. Jakupec, M. Galanski and B. K. Keppler, Inorg. Chem., 2013, 52, 8151-8162.

39 S. Theiner, C. Kornauth, H. P. Varbanov, M. Galanski, S. Van Schoonhoven, P. Heffeter, W. Berger, A. E. Egger and B. K. Keppler, Metallomics, 2015, 7, 1256-1264.

40 C. Korch, M. A. Spillman, T. A. Jackson, B. M. Jacobsen, S. K. Murphy, B. A. Lessey, V. C. Jordan and A. P. Bradford, Gynecol. Oncol., 2012, 127, 241-248.

41 C. Paton, J. Hellstrom, B. Paul, J. Woodhead and J. Hergt, J. Anal. At. Spectrom., 2011, 26, 2508-2518. 